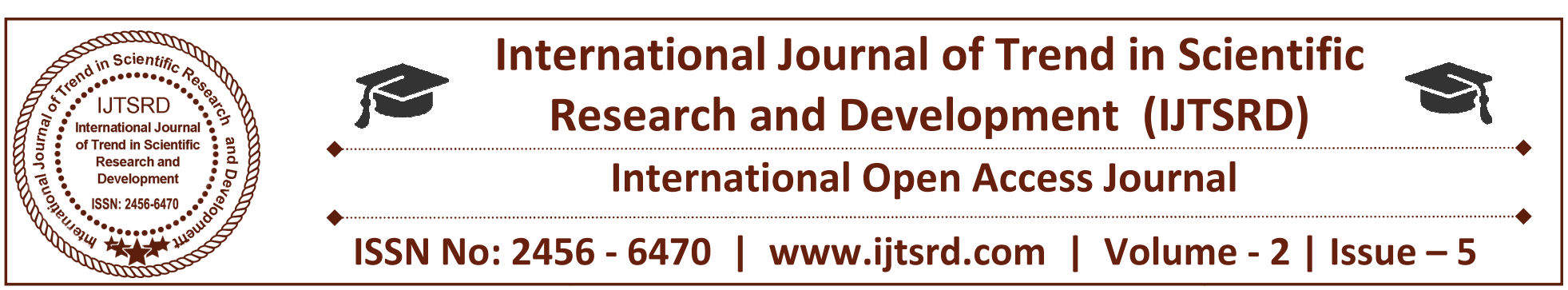

\title{
HDFS: Erasure-Coded Information Repository System for Hadoop Clusters
}

\author{
Ameena Anjum ${ }^{1}$, Prof. Shivleela Patil ${ }^{2}$ \\ ${ }^{1}$ Student, Department of Computer Science and Engineering, ${ }^{2}$ Associate Professor \& Head of Department, \\ Godutai Engineering College for Women Kalburgi, Karnataka, India
}

\section{ABSTRACT}

Existing disk based recorded stockpiling frameworks are insufficient for Hadoop groups because of the obliviousness of information copies and the guide decrease programming model. To handle this issue, a deletion coded information chronicled framework called HD-FS is developed for Hadoop bunches, where codes are utilized to file information copies in the Hadoop dispersed document framework or HDFS. Here there are two chronicled systems that HDFSGrouping and HDFS-Pipeline in HDFS to accelerate the information documented process. HDFS-Grouping is a Map Reduce-based information chronicling plan keeps every mapper's moderate yield Key-Value matches in a nearby key-esteem store and unions all the transitional key-esteem sets with a similar key into one single key-esteem combine, trailed by rearranging the single Key-Value match to reducers to create last equality squares. HDFS-Pipeline frames an information recorded pipeline utilizing numerous information hub in a Hadoop group. HDFS-Pipeline conveys the consolidated single key-esteem combine to an ensuing hub's nearby key-esteem store. Last hub in the pipeline is mindful to yield equality squares. HD-FS is executed in a true Hadoop group. The exploratory outcomes demonstrate that HDFSGrouping and HDFS-Pipeline accelerate Baseline's rearrange and diminish stages by a factor of 10 and 5, individually. At the point when square size is bigger than 32 M-B, HD-FS enhances the execution of HDFS-RA-ID and HDFS-EC by roughly 31.8 and 15.7 percent, separately.

Keywords: Hadoop distributed file system, replicabased storage clusters, archival performance, erasure codes, parallel encoding.

\section{INTRODUCTION}

Existing disk primarily based recorded storage frameworks square measure deficient for Hadoop teams owing to the symptom of data reproductions and also the guide decrease programming model. To handle this issue, associate degree obliteration coded data chronicled framework known as HD-FS is made, that documents uncommon need to data in expansive scale server farms to limit storage value. HD-FS utilizes parallel and pipelined cryptography procedures to accelerate data recorded execution in Hadoop circulated document framework on Hadoop teams. Specifically, HD-FS utilizes the tripartite data imitations and also the guide decrease pedagogy model in Hadoop bunch to assist the authentic execution in HD-FS, demonstrates to quicken the pedagogy procedure in HD-FS by the uprightness of data region of 3 reproductions of squares in HD-FS. The attendant 3 components spur to make up the deletion code base documented framework for Hadoop groups: a compressing need within the direction of bring down capability value, staggering expense adequacy of obliteration code storage and, the omnipresence of Hadoop process stages. Decreasing Storage charge Peta-bytes of data square measure lately place away in Brobdingnagian disseminated storage frameworks. With associate degree increasing range of capability hubs introduced, the data storage value goes up drastically. A large range of nodes ends up in a high risk of failures caused by unreliable elements, package glitches, machine reboots, maintenance operations and also the like. To ensure high responsibility and availableness within the presence of varied styles of failures, knowledge redundancy is often employed in cluster storage systems. 2 wide adopted fault tolerant solutions square measure replicating further 
knowledge blocks (i.e., 3X-replica redundancy) and storing further data as parity blocks (i.e., erasurecoded storage). as an example, the 3X-replica redundancy is utilized in Google's G-FS , HD-FS , Amazon S3 to attain fault tolerance. Also, erasurecoded storage is wide employed in cloud storage platform and knowledge centers. a technique to cut back storage value is to convert a $3 \mathrm{X}$-replicabased storage system into associate degree erasure-coded storage. It is sensible to take care of $3 \mathrm{X}$ replicas for often accessed knowledge. Significantly, managing non-popular knowledge mistreatment erasure coded schemes facilitates savings in storage capability while not adversely imposing performance penalty. A significant portion fof knowledge of knowledge of information $\}$ in knowledge centers square measure thought-about as non-popular data, as a result of knowledge have associate degree inevitable trend of decreasing access frequencies. Proof shows that the majority of information square measure accessed at intervals a brief length of the data's time period. as an example, over ninety p.c of accesses during a Yahoo!M45 Hadoop cluster occur with within the first day when knowledge creation. Applying Erasure Coded Storage. Though 3 X-replica redundancies achieves high performance than erasure-coded schemes, 3X-replica redundancy inevitably ends up in low storage utilization. Value effective erasure-coded storage systems square measure deployed in giant knowledge centers to attain high responsibility at low storage value.

\section{EXISTING SYSTEM}

Blast in huge information has prompted a surge in to a good degree substantial scale huge information investigation stages, transfer concerning increasing vitality prices. Large information figure show orders solid info space for procedure execution, and moves calculations to info. Leading edge cooling vitality administration procedures depend upon heat aware procedure occupation arrangement/relocation and square measure innately info position deist in nature It bodes well to stay up $3 \mathrm{X}$ reproductions for routinely ought to info. Peremptorily, overseeing non-famous info utilizing obliteration coded plans encourages reserve funds away limit while not antagonistically forcing execution penalty. A significant phase \{ of info of data of knowledge $\}$ in server farms square measure thought-about as non-prevalent information, since info have associate unavoidable pattern of decreasing access frequencies. Confirmation demonstrates that the overwhelming majority of data square measure gotten to within a quick length of the information's time period.

\section{DISADVANTAGES}

$>$ The Existing disk based mostly authentic warehousing frameworks area unit lacking for Hadoop teams thanks to the forgetfulness of knowledge reproductions and also the guide decrease programming model.

$>$ An intensive range of hubs prompts a high believability of disappointments caused by inconsistent components, programming glitches, machine reboots, support tasks and then forth. to confirm high unwavering quality and accessibility among the sight of various varieties of disappointments, data repetition area unit usually utilized as a vicinity of bunch warehousing frameworks.

\section{PROPOSED SYSTEM}

In this work, associate degree obliteration coded data chronicled framework referred to as HDFS for Hadoop bunches is planned. HDFS deals with varied Map errands over $\{$ the data the knowledge the data hubs that square measure chronicling their neighborhood information in equivalent. 2 chronicled plans square measure cleanly incorporated keen on HD-FS, that switch flanked by the 2 ways in lightweight of the scale and space of filed files. Middle of the road equality squares created within the Map expressions considerably bring down the $\mathrm{I} / \mathrm{O}$ and calculation weight amid the knowledge remake procedure. HD-FS lessens organize transfer within the middle of hubs over the span of data chronicling, in light-weight of the very fact that HD-FS take the good thing about the good data region of the $3 \mathrm{X}$-imitation system. It at that time be relevant the Map scale back instruction reproduction within the direction of build up the HD-FS framework, anyplace the 2 data recorded systems square measure actualised for Hadoop teams. We tend to produce HD-FS on the best purpose of Hadoop framework, facultative a framework head to effectively and speedily send our HD-FS while not modifying and revamp HD-FS in Hadoop teams. During this approach it directs broad trial to look at the results of the sq. dimension, file estimate, the esteem live on the execution of HD-FS.

\section{ADVANTAGES}

$>$ The handling of the knowledge is completed in parallel approach henceforward the results of the knowledge is fast and productive. 
$>$ Cheap product instrumentality is employed for the knowledge repositing reason, henceforward anyone will capable utilize this framework

$>$ The data misfortune and various things are taken care of by the framework itself.

\section{SYSTEM ARCHITECTURE}

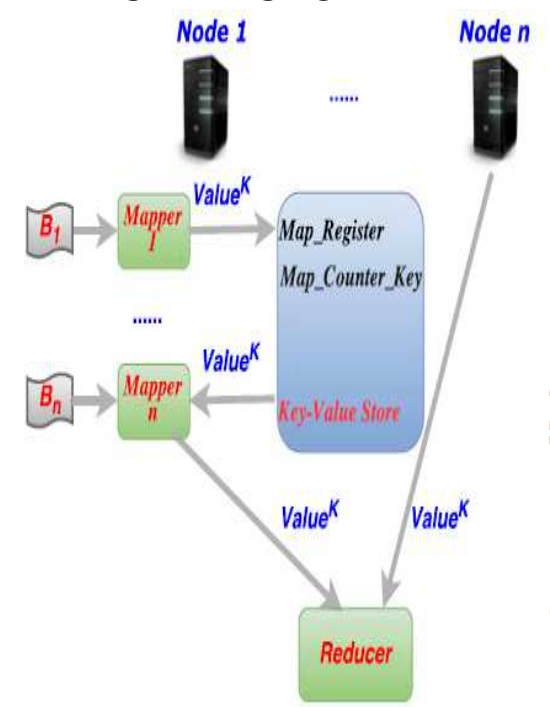

Fig 1: System Architecture

The HDFS system design follows the map scale back programming model however with economical thanks to store the fies within the system. It employs the map and scale back technique wherever the computer file is splitted into key worth pairs and so mapping is completed. After mapping the text worth combine is given as output, followed by partitioning and shuffling we tend to get the reduced output. Figure illustrates the Map Reduce-based strategy of grouping intermediate output from the multiple map pers. A standard knowledge is to deliver Associate in nursing intermediate result created by every plotter to a reducer through the shuffling part. To optimize the performance of our parallel archiving theme, we tend to cluster multiple intermediate results sharing an equivalent key into one Key-Value combine to be transferred to the reducer. Throughout the course of grouping, of course, the XOR operations square measure performed to come up with the worth within the Key-Value combine. The advantage of our new cluster strategy makes it potential to shift the grouping and computing load historically handled by reducers to map pers. In doing thus, we tend to alleviate the potential performance bottleneck drawback incurred within the reducer.

\section{MODULES}

1. HDFS Grouping - HD-FS Grouping combines every one the moderate key-esteem sets with a similar key into one single key-esteem match, trailed by shuffle the solitary Key-Value match in the direction of reducers to create final equality squares.

2. HD-FS Pipeline - It shapes an information documented pipeline utilizing different information hub in a Ha-doop group. HD-FS channel conveys the consolidated solitary keyesteem combine to an ensuing hub's neighborhood key-esteem store. Last hub in the pipeline is in charge of yielding equality squares.

3. Erasure Codes- To manage these disappointments, stockpiling frameworks depend on deletion codes. An eradication code adds excess to the framework to endure disappointments. The least difficult of these is replication, for example, RAID-1, where every byte of information is put away on two plates.

4. Replica-based capacity bunches -to diminish capacity cost is to change over a $3 \mathrm{X}$-replicabased capacity framework into a deletion coded capacity. It bodes well to keep up $3 \mathrm{X}$ reproductions for as often as possible got to information. Critically, overseeing non-prevalent information utilizing eradication coded plans encourages investment funds away limit without unfavorably forcing execution punishment.

5. Archival execution - To upgrade the execution of our parallel filing calculation, another gathering Strategy is utilized to diminish the quantity of moderate key-esteem sets. We likewise outline a neighborhood key-esteem store to limit the disk $\mathrm{I} / \mathrm{O}$ stack forced in the guide and diminish stages.

\section{INTERPRETATIONS OF RESULTS}

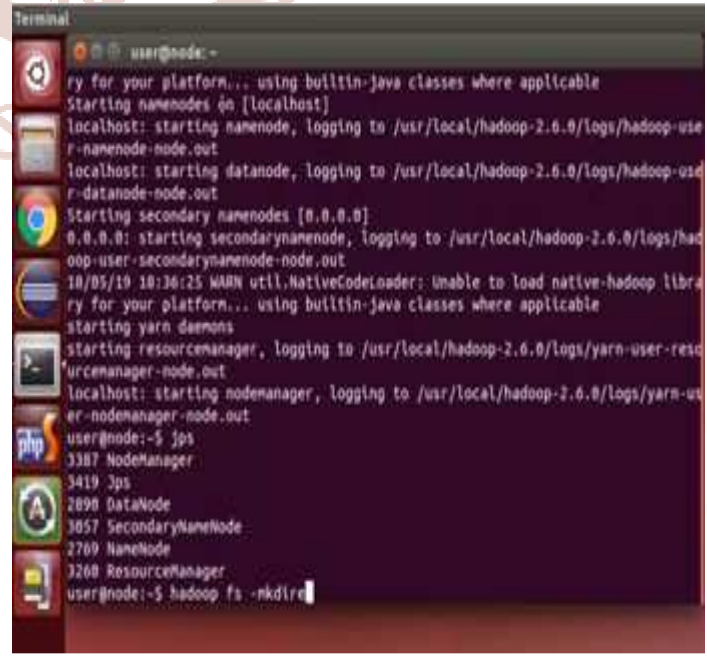

Fig 2: Here all the daemons in the hadoop cluster are started with the command start-all.sh. This command starts the name node, data node, secondary name node, resource manager. 
3rowse Directory

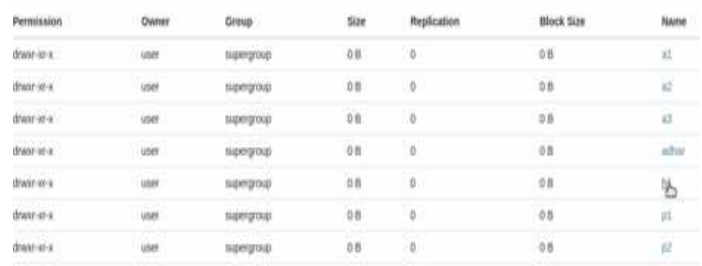

Fig 3: In the browse directory we get the detailed information of the file created in the cluster. single key-esteem combine on every hub. HD-FS Grouping exchanges the single key-esteem combine to a reducer, while HD-FS channel conveys this keyesteem match to the consequent hub in the chronicling channel. We actualized these two chronicling systems, which were thought about against the regular Map Reduce-based filing technique alluded to as Baseline. The test comes about demonstrate that HD-FS group and HD-FS channel can enhance the general documented execution of Baseline by a factor of 4 . Specifically, HD-FS group and HD-FS channel accelerate Baseline's shuffle and reduce stages by a factor of 10 and 5, independently.

\section{REFERENCES:}

1. J. Wang, P. Shang, and J. Yin, "Draw: another information gathering mindful information situation plot for information concentrated applications with intrigue territory," in Cloud Computing for Data-Intensive Applications. Berlin, Germany: Springer, 2014, pp. 149- 174.

2. S. Ghemawat, H. Gobi off, and S. - T. Leung, "The Google record framework," ACM SIGOPS Operating Syst. Rev., vol. 37, no. 5, pp. 29-43, 2003.

3. D. Borthakur, "The hadoop conveyed record framework: Architecture and plan, 2007," Apache Software Foundation, Forest Hill, MD, USA, 2012.

4. B. Calder, et al., "Windows sky blue stockpiling: A profoundly accessible distributed storage benefit with solid consistency," in Proc. 23rd ACM Symp. Working Syst. Standards, 2011, pp. $143-157$.

5. S. S. Mill operator, M. S. Shaalan, and L. E. Ross, "Journalist driven administration email framework utilizes message-reporter relationship information table for consequently connecting a solitary put away message with its journalists," U.S. Patent 6 615 241, Sep. 2, 2003.

\section{AUTHORS DETAILS}

\section{CONCLUSION}

In this manner it displays an eradication coded information recorded framework HDFS in the domain of Hadoop group processing. We future two filing techniques call HD-FS group and HDFS channel to accelerate authentic execution in Hadoop circulated file structure or HDFS. Both the chronicling plans embrace the Map Reduce base assembly procedure, which hush-up awake different middle of the road key-esteem sets contribution similar input keen on

\section{First author:}

AMEENA ANJUM completed her B.E from Poojya Doddappa Appa college of engineering pursuing M.Tech from Godutai engineering college for women kalburgi.

2. Second author:

SHIVALEELA PATIL Associate professor department computer science, Godutai engineering college for women kalburgi. 